\title{
DISKUSSIONSFORUM
}

\section{Desorientierung der TA oder Orientierungsgewinn?}

Einige Anmerkungen zum Vorschlag,
die TA hermeneutisch zu erweitern

von Knud Böhle, Institut für

Technikfolgenabschätzung und

Systemanalyse (ITAS), Karlsruhe

Gegenüber der von Armin Grunwald geforderten "hermeneutischen Wende" der TA angesichts spekulativer Technikzukünfte und kontroverser Debatten um die ,New and Emerging Sciences and Technologies" (NEST), wird hier für eine praxis- und prozessorientierte TA auch im Fall der NEST argumentiert. Die unterschiedliche Sicht folgt aus der jeweiligen Bestimmung der Ausgangssituation. Grunwald folgert aus dem Umstand, dass es bei den NEST kein robustes Wissen über ihre künftigen Folgen gibt, dass der TA nichts anderes übrig bliebe als sich hermeneutisch mit den visionären Narrativen der NEST und den zugehörigen Debatten zu befassen, um so doch noch Orientierungswissen zu generieren. Dieser Letzte-Ausweg-Ansatz blendet aus, so die Kritik, dass es zu dem Zeitpunkt, an dem TA sich mit einer der NEST zu befassen beginnt, schon manifeste soziotechnische Innovationsprozesse im Gang sind, es einen dazu gehörigen Sachstand der wissenschaftlich-technischen NEST-Entwicklung gibt, und auch schon Politiken, die auf visionäre Narrative rekurrieren. Eine an den laufenden Innovationsprozessen ansetzende TA, so die Gegenthese, sollte deshalb auch im Fall von NEST-Entwicklungen in der Lage sein, ohne Umwege politikrelevante Orientierungsleistungen zu erbringen.

Grunwald (2015) plädiert für eine ,hermeneutische Erweiterung der TA“, die - um das hier eingangs in aller Kürze zu rekapitulieren - folgendermaßen begründet wird (vgl. auch Grunwald 2014a; Grunwald 2014b): In Bezug auf die „New and Emerging Science and Technologies" (NEST) gibt es keine Möglichkeit robustes „Folgenwis- sen" zu generieren, das Politik und Gesellschaft orientieren könnte. Womit man zu tun habe, seien lediglich spekulative Zukunftserwartungen, Visionen oder Befürchtungen. Unter diesen Umständen sei eine „hermeneutische Wende“ (2015, S. 67) vorzunehmen, indem man sich den teils sehr lebhaften und kontroversen Debatten um NEST zuwendet und sie als Ausdruck unserer Gegenwart deutet. Hierbei sei einzubeziehen, ,was die Tatsache, dass diese Debatten heute stattfinden, über uns heute aussagt" (2015, S. 66). Die Divergenz der Zukünfte spiegele die Pluralität der Gegenwart, und diese sei nicht nur eine Pluralität der Werte, sondern auch eine der wissenschaftlichen wie der außerwissenschaftlichen Meinungen über epistemisch nicht klassifizierbare Zukunftsbilder. Deshalb sollen hermeneutische Untersuchungen zur Quelle der Generierung von Orientierung werden, wobei die erwartete Orientierungsleistung darin bestünde, ,aus Technikzukünften in ihrer Diversität etwas über uns, unsere gesellschaftlichen Praktiken, unterschwelligen Sorgen, impliziten Hoffnungen und Befürchtungen lernen zu können“ (2015, S. 68). Zur Durchführung solcher Untersuchungen müssten Sprachwissenschaften, hermeneutische Ansätze in Philosophie und Geisteswissenschaften, Kulturwissenschaften und auch die Hermeneutik in der Kunst ,an Bord der TA kommen“" (2015, S. 68). Dazu sei angemerkt: Die angeregte hermeneutische Erweiterung der TA ist gegenwärtig noch Desiderat, und die erhoffte Orientierungsleistung wurde bisher an keinem konkreten Fall erwiesen.

\section{Technology Assessment ist schon immer hermeneutisch gewesen}

Ohne Frage kann die Technikfolgenabschätzung von den Erkenntnissen einer Hermeneutik, die sich der Technik, der Zukunft und den Technikzukünften zuwendet, profitieren. Grunwald selbst (vgl. etwa 2012) und andere Philosophen (etwa Kaminski 2010 und neuerdings z. B. Gransche 2015; Kaiser 2015; Grübler 2015) haben dazu für die TA außerordentlich relevante Arbeiten verfasst. Das reicht aber nicht aus, um von der Notwendigkeit einer hermeneutischen Erweiterung der TA zu sprechen. TA hat schon immer das Wissen unterschiedlichster Diszipli- 
nen und Wissenschaftszweige einbeziehen müssen - Technikrecht, Governance- und Innovationsforschung, Wissenschaftsforschung, Risikoforschung, Techniksoziologie, hermeneutische Technikphilosophie, Technikgeschichte, Ethik etc. -, ohne dass daraus spezielle TA-Varianten hervorgegangen wären.

Auch ohne das Adjektiv „hermeneutisch“ zu führen, ist Technology Assessment in mehrfacher Hinsicht schon immer ,hermeneutisch“ gewesen. Der englische Ausdruck wird hier bewusst verwendet, um die in "Technikfolgenabschätzung“ mitgegebene „konsequentialistische“ Engführung auf „Folgen“ zu vermeiden. Technology Assessment ist dem gegenüber weiter gefasst und lässt sich allgemein als die Aufgabe verstehen, die Bedeutung vor allem neuer Technologien (sozial, sachlich, zeitlich) zu ermitteln und einzuschätzen. Dazu gehört es auch, die unterschiedlichen, durch Interessen, Ressourcen, Positionen und Werte geprägten Perspektiven einzunehmen und deutend zu verstehen. Dazu gehört ohne Frage auch, sich mit den Schriften und Werken, in denen diese Sichtweisen zum Ausdruck kommen, zu befassen. TA muss sich außerdem im Sinne eines „Know-how" darauf verstehen, „funktional bzw. akteursspezifisch differenzierte Perspektiven und Präferenzen in solche mit allgemeinen Geltungsanspruch zu transformieren" (Gloede 2007, S. 45) und eine Expertise zu erbringen, die von der Politik als anschlussfähig erachtet wird. Insofern TA in Debatten und Verständigungsprozesse involviert ist, ist auch hier Verstehen vorausgesetzt. Auch an der Generierung von Bedeutungen und Bedeutsamkeiten - Jung würde hier von, ,produktiver Hermeneutik“ sprechen (2012, S. 23) - ist TA beteiligt, wenn etwa durch TA-Beiträge zu Diskursen ein anderes Verständnis soziotechnischer Zusammenhänge entsteht oder neue Optionen im politischen Raum diskutiert werden. Anzumerken ist an dieser Stelle, dass wer sich für eine „hermeneutische Erweiterung der TA“ ausspricht, über die skizzierten Bezüge hinausgehen und explizieren sollte, von welcher philosophischen oder soziologischen Hermeneutik und welchen hermeneutischen Methoden (vgl. etwa Jung 2012; Hitzler/Honer 1997; Kurt/Herbrik 2014) er sich bei einer Erweiterung der TA etwas verspricht.

\section{Für ein Technology Assessment der NEST}

Im Folgenden soll kurz erläutert werden, dass ein Technology Assessment der NEST, trotz einiger Besonderheiten, ohne weiteres möglich erscheint. Technology Assessment (TA) lässt sich - kurz gefasst - als die wissenschaftliche Analyse von dynamischen und komplexen, soziotechnischen Konstellationen in Politik beratender Absicht verstehen. Das schlägt sich in Zuschnitt und Stil der Arbeiten nieder. Die politische Öffentlichkeit bildet den Entdeckungszusammenhang von Problematisierungen, die sich z. B. als ,hope, hype and fear" äußern und mit Erwartungen verbunden sind, dass die Probleme vom politischen System aufgegriffen und bearbeitet werden. TA ist dann ,,problemorientierte Forschung“ in Bezug auf diese im politischen Raum artikulierten Probleme. TA-Studien richten sich an Instanzen des politischen Systems und an die politische Öffentlichkeit. Ihre Ergebnisse sind als Beiträge zum politisch-öffentlichen Diskurs zu verstehen. Wissenschaftliche und technische Entwicklungen können in jeder Phase ihres Lebenszyklus problematisch und damit zum Gegenstand von TA werden, also auch im frühesten Stadium der Technikgenese bzw. im NEST-Zustand.

An drei zentralen Elementen lässt sich zeigen, dass die TA von NEST zwar Besonderheiten aufweist, aber trotzdem mit dem Standardinventar der TA bearbeitet werden kann:

1. Die Analyse kognitiv-praktischer Orientierungen in Innovationsprozessen ist für jede TA essentiell - unabhängig vom Stadium der Entwicklung und der konkreten Form des Orientierungswissens.

2. Da an den Innovationsprozessen, mit denen die TA sich befasst, lebende Akteure beteiligt sind, stehen über hermeneutische Text- und Medienanalysen hinaus, immer auch soziologische und partizipative Ansätze zur Verfügung, um die Bedeutung neuer Technologien zu ermitteln.

3. Auch bei den NEST ist es möglich, den wissenschaftlich-technischen Sachstand einzuholen.

\section{Ad 1) Kognitiv-praktische Orientierungen in Innovationsprozessen}

Inventions- und Innovationsprozesse (auch Transformationsprozesse) sind ohne kognitiv-praktische 
Orientierungen nicht denkbar. Das wusste schon die alte Leitbildforschung (Dierkes et al. 1992), es ist aber im Zuge der NEST-Debatten und des gleichzeitig aufkommenden „Vision Assessment“ wieder stärker ins Bewusstsein der TA-Community getreten. Das neu entfachte Interesse an der ,Zukunft“, den „Zukünften“, an Antizipation und ,,vorausschauendem Denken" tut ein Übriges dazu.

Kognitiv-praktische Orientierungen müssen aber nicht unbedingt Technikzukünfte sein. Es können auch, wie Jasanoff/Kim (2009) für den (herrschenden) Diskurs zur Atomenergie in den USA herausgearbeitet haben, recht starre Ideologeme als leitende „socio-technical imaginaries“ auftreten. Im Falle ,neuer“ Technologien dürften häufig Prospektionen und Antizipationen vorliegen. Bemerkenswert sind Anzeichen in der Forschung, stärker als bisher auf die Orientierungsfunktion nicht evidenten Wissens in unterschiedlichen Praxisfeldern zu achten. Für die Ökonomie hat z. B. Beckert die Bedeutung fiktionaler Erwartungen (Beckert 2013) herausgearbeitet. Möglicherweise wirken selbst konsequentialistisch auftretende Technikzukünfte, wie Prognosen oder Modelle, nicht deshalb, weil ihr Wissen evident wäre, sondern weil die gewählte Wissensform in der Lage ist, Geltungsansprüche zu transportieren, Unsicherheit zu reduzieren und Entscheidungen zu legitimieren (vgl. etwa Scheer 2013 für Computersimulationen und Dieckhoff 2015 für Klimamodelle und -szenarien).

Wenn es so ist, dass die Handlungsfähigkeit in der Gegenwart durch Annahmen über die $\mathrm{Zu}$ kunft, z. B. in Form von Technikzukünften (Prognosen, Modelle, visionäre Narrative etc.), hergestellt wird, dann ist die Frage zentral, wie diese Formen in der Innovationspraxis, zu der auch die politische Praxis gehört, wirken. Die Wirkung visionärer Narrative in Innovationsprozessen zu erforschen, ist da nur ein spezieller Fall.

Orientierendes Wissen, egal in welcher Form, tritt nicht von außen hinzu, sondern entsteht in der Praxis, in der es verwendet wird (vgl. zu einer „epistemology of practice“ Cook/Wagenaar 2012). Im einfachsten Fall, auf den Burg (2014) (in ihrer Antwort auf Nordmann 2014) abstellt, geht es um die praktisch notwendige Rolle von Antizipationen im Forschungsprozess: „they help to give meaning to present research and development actions, based on imaginations of the actions that could follow them" (2014, S. 101). Die Überlegungen von Schulz-Schaeffer (2013) zur Orientierung von Technikentwicklung durch Szenarien entsprechen dem, ebenso wie die Untersuchungen von Lösch/Schneider (2015 i. E.) zur Funktion der Smart-Grid-Visionen in Realexperimenten im Kontext der Energiewende oder die Beobachtung der Wirksamkeit ,konkretutopischer" Orientierung in Open-Design-Entwicklergruppen (Schneider 2014). Zukunftsvorstellungen interessieren die TA mithin eben nicht nur hermeneutisch - bezogen auf das, was als wünschenswert und machbar vorgestellt wird -, sondern in einer funktionalen Perspektive auch dahingehend, was sie ermöglichen bzw. welche Handlungs- und Koordinationsprobleme in Innovationsprozessen sie zu bewältigen helfen.

\section{Ad 2) Empirische Sozialforschung für die Analyse soziotechnischer Konstellationen}

Die Analyse soziotechnischer Innovationsprozesse in Politik beratender Absicht impliziert die Untersuchung der Multiperspektivität von Interessen und Präferenzen und der kognitiv-praktischen Orientierungen (Visionen, Leitbilder, Weltanschauungen $u$. a.), die dabei eine Rolle spielen. Wenn in der politischen Öffentlichkeit Nachfrage nach TA entsteht, ist im Normalfall immer schon auswertbares Wissen vorhanden, weil über die zu untersuchenden Phänomene oder Probleme schon Studien vorliegen, weil es Strategiepapiere gibt, Lobbys und NGOs Stellungsnahmen erarbeitet und die Medien das Thema aufgegriffen haben, kurzum ein öffentlicher Diskurs bereits im Gange ist. Diesen Diskurs zu kennen, ist unumgänglich. Dazu verhelfen Diskursanalysen, Medienanalysen, Argumentationskartierungen, Synopsen und sicherlich auch hermeneutische Analysen.

Häufig dürfte die Rekonstruktion des Diskurses aber nicht ausreichen, weil das für ein Assessment in Politik beratender Absicht benötigte Wissen noch gar nicht in ausreichendem Maße explizit vorhanden ist. Die multiplen Perspektiven, Präferenzen und Interessen in soziotechnischen Konstellationen liegen noch gar nicht artikuliert vor, und müssen folglich erst mittels sozialwissenschaftlicher Methoden erhoben werden. Repräsentative Umfragen, Experteninterviews, Delphi-Befragun- 
gen, Fokusgruppen, Workshops, teilnehmende Beobachtung und anderes mehr können hier zum Einsatz kommen. Diese Methoden stehen auch zur Untersuchungen der NEST zur Verfügung, denn selbst wenn man annähme, dass noch nicht viel an Technik vorhanden ist, an relevanten Akteuren fehlt es nicht. Die Analyse visionärer Narrative muss sich nicht darauf beschränken, in klassisch hermeneutischer Weise den Sinn von Texten und Werken abwesender Autoren zu interpretieren. Die am Innovationsprozess beteiligten Akteure stehen (trotz möglicher Probleme des Feldzugangs) zur Verfügung und es besteht die Möglichkeit, mit ihnen zu kommunizieren und darüber auswertbares Material und neue Einsichten über den Innovationsprozess zu gewinnen.

\section{Ad 3) Sachstand der NEST}

TA muss sich mit den konkreten wissenschaftlichen Entwicklungen, Artefakten und technischen Systemen befassen, und den naturwissenschaftlichen und technischen Sachstand einholen, um eine Einschätzung des heute und zukünftig Machbaren zu gewinnen. Es gehört zu den wichtigen Aufgaben der TA, hochfliegende Erwartungen durch Konfrontation mit dem Sachstand auf das in absehbarer Zeit Mögliche zu reduzieren und dadurch zur politischen und gesellschaftlichen Orientierung beizutragen. In dem Zusammenhang wird häufiger von einem „reality check“ gesprochen. Dieser „reality check" erfolgt sowohl über Dokumentenanalysen (unter anderem auch Patentanalysen oder bibliometrische Publikationsauswertungen) als auch mittels sozialwissenschaftlicher Methoden wie Experteninterviews oder Delphi-Befragungen, die in der Sache kompetente Wissenschaftlerinnen und Wissenschaftler dazu veranlassen sollen, ihre Einschätzungen mitzuteilen. Fachwissenschaftler im Projektteam, Gutachten von Experten oder die längerfristig angelegte Teilnahme von TA-Forschern in entsprechenden Forschungseinrichtungen bieten sich als zusätzliche Wege an, den Sachstand zu ermitteln. Bezogen auf visionäre Narrative dürfte gerade der „reality check“ der TA dazu beitragen, überzogene Erwartungen abzubauen und dadurch politische Handlungsmöglichkeiten zu eröffnen. Beispiele sind etwa die Studien des TAB zur Nanotechnologie (Paschen et al. 2004) oder zum ko- gnitiven Enhancement (Sauter/Gerlinger 2012). In einzelnen Fällen mag der „reality check“ sogar dazu beitragen haben, Zukunftstechniken zu ,normalisieren“ und „regulierbar" zu machen (vgl. Grunwald/Hocke 2010; s. auch Grunwald 2014a, S. 278, der von der ,normalization of nanotechnology" spricht, und Kaiser 2015, S. 144f., der, bezogen auf den TAB-Bericht zur Nanotechnologie, von der „Regulierbarmachung“ durch Technikfolgenabschätzung ausgeht). Nicht nur das visionäre Narrativ, sondern auch den Sachstand und seine wahrscheinliche Weiterentwicklung in den nächsten Jahren zu kennen, erscheint unverzichtbar für eine TA der NEST.

\section{Ein Fazit: Orientierung durch ein Vision Assessment der NEST}

Die Annahme Grunwalds, dass es hermeneutischer Untersuchungen von NEST-Debatten bedürfe, um Orientierungswissen zu gewinnen, weil Prognosen oder robuste Szenarien als „konsequentialistisch" Orientierung gebende Technikzukünfte dafür nicht zur Verfügung stünden, ist nicht einfach nachzuvollziehen. Zum einen wird ein Verständnis der „hermeneutischen Erweiterung der TA" dadurch erschwert, dass nicht erläutert wird, mit welcher philosophischen oder soziologischen Hermeneutik und welchen hermeneutischen Methoden das Ziel erreicht werden soll.

Zum anderen, und das ist für den Hinweis auf eine mögliche Desorientierung der TA ausschlaggebend, ist der „Letzte-Ausweg-Ansatz“ Grunwalds irreführend. Er besagt: Weil man über die Folgen der NEST nichts direkt sagen könne, gelte es, die sie begleitenden Visionen hermeneutisch zu untersuchen. Über Folgen der NEST lässt sich zwar in der Tat nicht viel sagen. Aber auch im Fall der NEST - so die Gegenthese - greifen die klassischen Verfahren der TA, nämlich Sachstandsanalyse (,reality check"), Konstellations- und Wirkungsanalysen sowie Politik- und GovernanceAnalysen, um Wissen zu generieren, das der Politik und der Öffentlichkeit hilft, sich zu orientieren.

Der „Letzte-Ausweg-Ansatz“ ist zudem historisch wenig überzeugend. Wenn NEST-Technologien, so sehr sie auch noch in den Anfängen stecken mögen, öffentliche Aufmerksamkeit erregen, also als „hype, hope and fear-Technologien“ 
wahrgenommen werden, und schließlich TA auf den Plan gerufen wird, ist immer schon sehr viel passiert. Denkt man beispielsweise an die Nanotechnologie, so wurde die Vision spätestens Mitte der 1980er Jahre (Drexler 1986) formuliert (vgl. zur Chronologie dieser Vision Simakova/Coenen 2013, S. 244ff.). Die erste TA-Studie dazu wurde in Deutschland aber erst im Jahr 2003 durch das TAB abgeschlossen (Paschen et al. 2004). Zu dem Zeitpunkt hatte sich auch die Politik schon durch entsprechende Förderprogramme positioniert, die ersten Nanoprodukte waren bereits auf dem Markt. Es gab also durchaus Konstellationen und Prozesse, die man untersuchen konnte und vor allem auch viele Personen, die man befragen konnte. Sieht man sich die TAB-Studie zu dieser NEST-Technologie genauer an, wird deutlich, dass sie weitgehend dem Ansatz einer Konstellations- und Innovationsprozessanalyse entspricht.

Außerdem sollte man sich vor Augen halten, dass die NEST nicht nur eine Vorgeschichte haben, sondern die weitere Entwicklung der NEST und ihre gesellschaftliche Einbettung langfristige Prozesse sind. Denkt man an ,alte“ NEST wie die Kernforschung oder die Künstliche Intelligenz, die trotz ihrer mehr als 50-jährigen Geschichte virulent bleiben mit Themen wie Transmutation, Fusion, humanoiden Robotern und ,Superintelligenz", wird deutlich, dass auch die neueren NEST wie „Synthetische Biologie“ oder Gentechnik die TA langfristig beschäftigen werden.

Die Zurückweisung des „Letzte-AuswegAnsatzes" und die abwartende Haltung gegenüber einer „hermeneutischen TA“ drückt keine Geringschätzung der Bedeutung kognitiv-praktischer Orientierungen in Innovationsprozessen aus. Im Gegenteil: Eine praxis- und prozessorientierte TA bietet die Möglichkeit, visionäre Narrative nicht nur a) als auszulegende Texte zu verstehen, sondern b) auch als Praktiken in Innovationsprozessen (z. B. Visioneering) zu begreifen, des weiteren c) ihr dynamisches Wechselverhältnis mit dem wissenschaftlich-technischen Sachstand zu verfolgen, und d) ihre Funktionen im Innovationsprozess - z. B. der Motivation, Handlungskoordination, Entscheidungsermöglichung - herauszuarbeiten. Die genannten Punkte können als Eckpunkte eines nicht nur hermeneutischen „Vision Assessments“ dienen.
Die Forderung Grunwalds (2015, S. 68; Grunwald 2014a, S. 276), dass den Prozessen der Bedeutungszuschreibung in frühen Phasen der Technologieentwicklung im Rahmen der TA mehr Beachtung geschenkt werden sollte, ist zuzustimmen. Selbst Konzepte wie "Synthetische Biologie" oder Nanotechnologie sind nicht voraussetzungslos. Die Konzepte selbst erscheinen bereits als Ergebnis einer sinnstiftenden Praxis, eines „Visioneering“ (vgl. auch McCray 2012), das sich in den Forschungs- und Förderlandschaften niedergeschlagen hat und dort wirksam ist.

Als wissenschaftlichen Referenzrahmen für entsprechende Untersuchungen zum „Vision Assessment" würde ich (ähnlich wie Grunwald) die Wissenssoziologie und mit ihr die Diskursforschung an erster Stelle sehen, wenn sie den Kampf um Bedeutungen im Kontext einer Praxis- und Prozessforschung ansiedelt (wie in Braun 2014 für die politische Ebene skizziert) und wenn sie auch die funktionale Seite von Wissensformen bezogen auf Innovations- und Transformationsprozesse adressiert. Weitere Fragen, die für die TA im Zusammenhang mit den NEST interessant sind, kann die Wissenssoziologie in den meisten Fällen nicht allein, sondern nur interdisziplinär, beantworten:

- Mit welchen Wissensformen bzw. Wissensobjekten (Prognosen, Modelle, Visionen etc.) und mittels welcher Praktiken werden an der Schnittstelle von Wissenschaft und Politik heutzutage typischerweise Entscheidungsprozesse unterstützt?

- Welche Funktionen der Handlungs- und Entscheidungsermöglichung sowie der Handlungskoordination können spekulative Technikzukünfte manifest oder latent erfüllen?

- Folgen die NEST-Visionen einem bestimmten Muster, etwa als Versprechung eines ,technology fix" für soziale Probleme? Stellen sie bloß aktuelle Formen eines überkommenen szientistischen Weltbildes dar?

- Ist Grundlagenforschung, die staatlich gefördert werden will, heutzutage visionspflichtig geworden, d. h. gezwungen ihren künftigen gesellschaftlichen Nutzen in Form von Visionen zu artikulieren?

- Inwieweit geht mit den NEST eine Transformation der Wissenschaft einher, wie etwa 
Nordmann (2005; Nordmann 2011) annimmt? Gilt das für alle NEST?

- Wenn die strategische Produktion von Visionen und dazugehörigen Bedürfnissen heutzutage als „Visioneering“ erfolgt, in welchen Abteilungen, Denkfabriken oder Zukunftswerkstätten werden sie wie produziert?

- In welchem Sinne ist TA selbst eine Visionen verändernde Praxis?

Ich würde vermuten, dass Armin Grunwald wenig gegen diese Fragen einzuwenden hat - auch wenn sie nicht auf Sinnverstehen, sondern auf den Strukturwandel von Wissenschaft, Politik und Wirtschaft im Zusammenhang mit Innovationsprozessen abzielen.

\section{Danksagung}

Ich danke der Kollegin Arianna Ferrari und den Kollegen Reinhard Heil, Andreas Lösch, Ulrich Riehm, Christoph Schneider und Klaus Wiegerling für ihre Anmerkungen zu einer früheren Fassung dieses Diskussionsbeitrags.

\section{Literatur}

Beckert, J., 2013: Imagined Futures: Fictional Expectations in the Economy. In: Theory and Society $42 / 3$ (2013), S. 219-240

Braun, K., 2014: Im Kampf um Bedeutung: Diskurstheorie und Diskursanalyse in der interpretativen Policy Analyse. In: Zeitschrift für Diskursforschung 01 (2014), S. 77-101

Burg, S. van der, 2014: On the Hermeneutic Need for Future Anticipation. In: Journal of Responsible Innovation 1/1 (2014), S. 99-102

Cook, S.D.N; Wagenaar, H., 2012: Navigating the Eternally Unfolding Present. Toward an Epistemology of Practice. In: The American Review of Public Administration 42/I (2012), S. 3-38

Dieckhoff, Chr., 2015: Modellierte Zukunft - Energieszenarien in der wissenschaftlichen Politikberatung. Bielefeld

Dierkes, M.; Hoffmann, U.; Marz, L., 1992: Leitbild und Technik. Zur Genese und Steuerung technischer Innovationen. Berlin

Drexler, E.K., 1986: Engines of Creation. Garden City, N.Y.
Gloede, F., 2007: Unfolgsame Folgen. Begründungen und Implikationen der Fokussierung auf Nebenfolgen bei TA. In: Technikfolgenabschätzung - Theorie und Praxis 16/1 (2007), S. 45-54

Gransche, B., 2015: Vorausschauendes Denken. Philosophie und Zukunftsforschung jenseits von Statistik und Kalkül. Bielefeld

Grübler, G., 2015: Wissenschaft, Moral und Heil. Der Horizont moralischer Phänomene und die Arbeit am Vertrauen in die Welt. Würzburg

Grunwald, A., 2012: Technikzukünfte als Medium von Zukunftsdebatten und Technikgestaltung. Karlsruhe

Grunwald, A., 2014a: The Hermeneutic Side of Responsible Research and Innovation. In: Journal of Responsible Innovation 3/1 (2014), S. 274-291

Grunwald, A., 2014b: Technikfolgenabschätzung als „Assessment" von Debatten: TA jenseits der Technikfolgenforschung. In: Technikfolgenabschätzung Theorie und Praxis 23/2 (2014), S. 9-15

Grunwald, A., 2015: Die hermeneutische Erweiterung der Technikfolgenabschätzung. In: Technikfolgenabschätzung - Theorie und Praxis 24/2 (2015), S. 65-69 Grunwald, A.; Hocke, P., 2010: The Risk Debate on Nanoparticles: Contribution to a Normalisation of the Science/Society Relationship? In: Rehmann-Sutter, C.; Maasen, S.; Kurath, M. et al. (Hg.): Governing Future Technologies. Nanotechnology and the Rise of an Assessment Regime. Dodrecht, S. 157-177

Hitzler, R.; Honer, A., 1997: Hermeneutik in der deutschsprachigen Soziologie heute. In: Hitzler, R.; Honer, A. (Hg.): Sozialwissenschaftliche Hermeneutik. Opladen, S. 7-27

Jasanoff, S.; Kim, S.-H., 2009: Containing the Atom: Sociotechnical Imaginaries and Nuclear Power in the United States and South Korea. Minerva 47, S. 119-146 Jung, M., 2012: Hermeneutik zur Einführung. Vierte vollständig überarbeitete Auflage. Hamburg

Kaiser, M., 2015: Über Folgen. Technische Zukunft und politische Gegenwart. Weilerswist

Kaminski, A., 2010: Technik als Erwartung. Grundzüge einer allgemeinen Technikphilosophie. Bielefeld Kurt, R.; Herbrik, R., 2014: Sozialwissenschaftliche Hermeneutik. In Nina Baur, N.; Blasius, J.: Handbuch Methoden der empirischen Sozialforschung, Wiesbaden, S. 473-491

Lösch, A; Schneider Chr., 2015 (i. E.): Experimental Re-arrangements: The Smart Grid and the Power/ Knowledge Apparatus of the German Energy Transition. Special Issue: Energy, Society and Culture. In: Innovation: The European Journal of Social Science Research 
McCray, P., 2012: The Visioneers: How a Group of Elite Scientists Pursued Space Colonies, Nanotechnologies, and a Limitless Future, Princeton

Nordmann, A., 2005: Was ist TechnoWissenschaft? Zum Wandel der Wissenschaftskultur am Beispiel der Nanoforschung und Bionik. Berlin, S. 1-10

Nordmann, A., 2011: The Age of Technoscience. In: Nordmann, A.; Radder, H.; Schiemann, G. (Hg.): Science Transformed? Debating Claims of an Epochal Break. Pittsburgh, S. 19-30

Nordmann, A., 2014: Responsible Innovation, the Art and Craft of Anticipation. In: Journal of Responsible Innovation 1/1 (2014), S. 87-98

Paschen, H.; Coenen, Chr.; Fleischer, T. et al., 2004: Nanotechnologie. Forschung, Entwicklung, Anwendung. Berlin

Sauter, A.; Gerlinger, K., 2012: Der pharmakologisch verbesserte Mensch. Leistungssteigernde Mittel als gesellschaftliche Herausforderung. Berlin

Scheer, D., 2013: Computersimulationen in politischen Entscheidungsprozessen: Zur Politikrelevanz von Simulationswissen am Beispiel der $\mathrm{CO}_{2}$-Speicherung. Wiesbaden

Schneider, Chr., 2014: Laser Cutter trifft Ernst Bloch: Open Design als konkrete Utopie. In: Korczak, D. (Hg.): Visionen statt Illusionen. Wie wollen wir leben? Kröning, S. 123-136

Schulz-Schaeffer, I., 2013: Scenarios as Patterns of Orientation in Technology Development and Technology Assessment. Outline of a Research Program. In: STI Studies 9/1 (2013); http://www.sti-studies.de/ojs/index. php/sti/article/download/129/97 (download 6.10.15)

Simakova, E.; Coenen, Chr., 2013: Visions, Hype, and Expectations: A Place for Responsibility. In: Owen, R.; Heintz, M.; Bessant, J. (Hg.): Responsible Innovation: Managing the Responsible Emergence of Science and Innovation in Society. Chichester, S. 241-266

\section{Kontakt}

Dipl.-Soz. Knud Böhle, M.A.

Institut für Technikfolgenabschätzung und

Systemanalyse (ITAS)

Karlsruher Institut für Technologie (KIT)

Karlstraße 11, 76133 Karlsruhe

Tel.: +49 721 608-22989

E-Mail: knud.boehle@kit.edu

\section{English Stammer}

Eine Polemik in 12 Bildern

\section{von Marcus Steierwald, Universität Tübingen}

Die Kernfrage dieser Ausführungen lautet: Werden durch den Vorrang des Englischen die Fundamente der wissenschaftlichen Erkenntnis in den TA-Wissenschaften, nämlich Diskurs und interdisziplinäres Arbeiten, gefestigt oder zerstört? Faktum ist: Die Wissenschaft bedient sich zunehmend der englischen Sprache. Da wir überwiegend keine muttersprachlich Englisch Sprechenden (MES) sind, ist es interessant, einmal die Effizienz eines Wechsels in diese Fremdsprache anzuschauen. Wir tun das hier in 12 Bildern, die manchmal luzide angelegt sind, aber hoffentlich stets unterhaltsam. Anders gesagt: The science helps itself increasingly of the English language. Because we are predominantly none native-language English speaking (MES), it is interesting to look (watch) once at the efficiency of a change into this foreign language. We act here in 12 pictures which are sometimes put on (invested) luzide, but hopefully always entertaining (amusing) ${ }^{1}$.

\section{Englisch schafft Frieden und Freunde}

Eines Tages traf der Autor bei einem Sommerfest der LMU einen Kollegen aus dem Kongo. Wir unterhielten uns großartig per Französisch, Englisch und winzigen Brocken Italienisch, Lateinisch und Deutsch. Anschließend war die Welt ein Stückchen vertrauter und verständlicher geworden.

Auch das Friedenschaffen ist viel einfacher, wenn wir einander schon sprachlich verstehen. Denn was bedeutet es, wenn der Mensch, der mir mit einer Waffe entgegentritt, brüllt brudij skrakodin, njemesch alpusli? Meint er nun Bruder, sei friedlich, wir können reden! oder brutaler Schurke, jetzt bist du hin...? In solchen Fällen ist die Verwendung einer gemeinsamen Fremdsprache sehr anzuraten, so das Pensionsalter gesund erreicht werden soll.

Die Babylonische Sprachverwirrung begründete die Tradition der Sehnsucht nach einer Weltsprache, die Frieden und Harmonie ermöglicht (vgl. Moses 1000 v. Chr., Kap. 11, 1-9). 